\title{
Peri- und intraoperative Gerinnungsstörungen und ihre Therapieempfehlungen
}

\author{
Wolfgang Korte \\ Zentrum für Hämostaseologie, Zentrum für Labormedizin St. Gallen, St. Gallen, Schweiz
}

\section{Schlüsselwörter \\ Gerinnung · Blutung · Operation · Geburt · Trauma}

\section{Zusammenfassung}

Hintergrund: Aufgrund neuer diagnostischer Technologien und der zunehmenden Verfügbarkeit von Gerinnungsfaktorkonzentraten hat die perioperative Hämostase in den letzten Jahren zunehmende Aufmerksamkeit erhalten. Methode: Die Literatur wurde im Hinblick auf die Diagnostik, Pathophysiologie und Behandlungsmöglichkeiten von peri- und intraoperativen Gerinnungsstörungen sowie bezüglich eigener Erfahrungen in den verschiedenen erwähnten Bereichen durchsucht. Ergebnisse und Schlussfolgerungen: Peri- und intraoperative Hämostasestörungen sind nicht selten. Sie sind in der Regel mit einem strukturierten diagnostischen Vorgehen klinisch einfach zu erfassen. Mittlerweile können innerhalb der unterschiedlichen klinischen Entitäten verschiedene zugrunde liegende Pathophysiologien identifiziert werden; damit können auch die entsprechenden, zum Teil unterschiedlichen Behandlungsmethoden zum Einsatz gebracht werden. Die kontinuierliche Beobachtung der klinischen Situation zusammen mit unter Umständen wiederholten Laboruntersuchungen erlaubt die frühzeitige Erfassung und Behandlung.

\section{Einleitung}

Das perioperative Hämostasemanagement hat in den letzten Jahren große Fortschritte gemacht. Dies ist wahrscheinlich auch darauf zurückzuführen, dass das Interesse an der perioperativen Hämostase, möglicherweise im Rahmen des demographischen Wandels, zugenommen hat: Die Menschen werden älter und bedürfen daher fortgesetzter und verstärkter medizinischer Versorgung, einschließlich - immer kom-

\author{
Keywords \\ Coagulation · Bleeding · Surgery · Childbirth · Trauma
}

\section{Summary \\ Peri- and Intraoperative Coagulopathies and Their \\ Treatment}

Background: Due to new diagnostic technologies and the increasing availability of coagulation factor concentrates, peri- and intraoperative bleeding has received increasing attention over the last years. Method: The literature was reviewed regarding diagnostics, pathophysiology, treatment modalities as well as own experiences gathered concerning these aspects. Results and Conclusions: Peri- and intraoperative coagulopathies are frequently encountered. They can be detected with simple, structured diagnostic approaches based on the clinical presentation. Different pathophysiologies have been detected in different clinical entities of coagulopathies. This allows the use of different, specific treatment modalities. Continued observation of the clinical presentation, together with repetitive laboratory evaluations, allows an early detection and treatment.

\begin{tabular}{ll}
\hline KARGER & ( ) 2013 S. Karger GmbH, Freiburg \\
1662-6664/13/0295-0303\$38.00/0 \\
$\begin{array}{l}\text { Fax +49 761452 07 14 } \\
\begin{array}{l}\text { Information@Karger.com } \\
\text { www.karger.com }\end{array}\end{array}$ & $\begin{array}{l}\text { Accessible online at: } \\
\text { www.karger.com/vim }\end{array}$
\end{tabular}


Trotz dieser Notwendigkeit gibt es bis heute keinen quasi universalen Konsens, wie perioperative Blutungen zu verhindern sind oder wie man sich diesem Problem nähern sollte. Dies ist möglicherweise auch ein Ausdruck der Heterogenität des Problems. Die Aufgabe dieses Artikels ist daher, einen Überblick über die verschiedenen Möglichkeiten zu geben, wie das Problem peri- und intraoperativer Blutungen angegangen werden kann. Dabei wird auf die Definition und Quantifizierung von koagulopathischen Blutungen während der Operation, mögliche Unterschiede der Pathophysiologie bei verschiedenen Arten von Operationen, die verschiedenen verfügbaren Behandlungsmöglichkeiten und die möglichen unterschiedlichen Ziele, die mit den unterschiedlichen Behandlungsstrategien erreicht werden sollen, eingegangen.

\section{Definition und Quantifizierung von intraoperativen Blutungen und Gerinnungsstörungen}

Historisch werden intraoperative Blutungen, die stärker als erwartet erscheinen oder die mit den üblichen lokalen Maßnahmen nicht adäquat kontrolliert werden können, als koagulopathische Blutungen interpretiert. Die Schwierigkeit liegt dabei nicht selten in der Problematik der Unterscheidung zwischen vermehrter Blutung aufgrund einer größeren Verletzung (also de facto des Zusammenbruchs einer mechanischen Barriere) und einer protrahierten Blutung bei gleichzeitig veränderter Zusammensetzung des Blutes (sodass die normalerweise bestehende Kompensationsfähigkeit des Systems für kleinere Verletzungen aufgehoben ist, d.h. Koagulopathie). Wie sich aus dieser Beschreibung ergibt, ist es unwahrscheinlich, dass nur der eine (größere Verletzung) oder der andere Grund (Koagulopathie) als einzige Ursache einer vermehrten intraoperativen Blutung identifiziert werden kann. Obwohl diese Überlegungen offensichtlich und einfach erscheinen, ist es wichtig, sich diese grundlegenden Prinzipien bei einer übermäßigen intraoperativen Blutung wiederholt vor Augen zu führen. Denn: Der Grund für eine übermäßige Blutung kann sich intraoperativ verändern, und es ergibt sich logischerweise, dass bei von Natur aus unterschiedlichen Pathophysiologien auch unterschiedliche Therapieansätze zum Tragen kommen müssen. Es ist daher notwendig, diese Unterscheidung bei jedem einzelnen übermäßigen Blutungsgeschehen jeweils erneut zu treffen, um daraus den notwendigen Therapieansatz (Wiederherstellung der mechanischen Barriere vs. Verbesserung der veränderten Blutzusammensetzung) abzuleiten.

Der derzeitige Ansatz zum Erkennen vermehrter intraoperativer, koagulopathisch bedingter Blutungen ist in der Regel die visuelle Kontrolle des operativen Bereichs mit Erkennung einer «diffusen Blutung ohne sichtbares Gerinnsel» [1]. Da intraoperative Blutungen sehr schnell auftreten können und nicht selten nur eines der Ereignisse sind, die Aufmerksamkeit benötigen, ist es offensichtlich, dass die Identifikation der zugrunde liegenden Pathophysiologie schnell, einfach und möglichst zuverlässig erfolgen muss. Wir haben vorgeschlagen, dass die klinische Identifikation einer koagulopathischen Blutungsneigung intraoperativ in der Regel durch die Beantwortung von drei einfachen Fragen bzw. Feststellungen erfolgen kann:

- War der betroffene Bereich, in dem mikrovaskuläre Blutungen wahrgenommen werden, vorher «trocken»?

- Es sind keine anderen angeborenen oder präoperativ auffälligen Gerinnungsstörungen bekannt.

- Es liegen keine Hinweise für «mechanisch (d.h. durch Gefäßverletzungen) bedingte» Blutungen vor.

Wenn alle diese Fragen bzw. Aussagen bejaht werden können, dann kann vom Vorliegen einer neu aufgetretenen Koagulopathie ausgegangen werden. Dieser validierte [2] Ansatz erlaubt eine strukturierte klinische Diagnose einer erworbenen intraoperativen Koagulopathie.

Wichtig ist auch zu erkennen, dass jede mechanische Blutung, die mittels Volumenunterstützung und Blutprodukten über längere Zeit behandelt wird, aufgrund des damit verbundenen Verdünnungseffekts und durch den Verbrauch von Gerinnungsfaktoren bei genügend langer Dauer auch zu einer koagulopathischen Blutung wird [3, 4].

Neben der Identifikation einer intraoperativen Koagulopathie ist es im Verlauf einer Operation auch wichtig, die biologischen Wirkungen dieser Pathologie quantifizieren $\mathrm{zu}$ können, wie z.B. den Blutverlust, die Komplikationsrate, die Verwendung von Blutprodukten usw.

Die Quantifizierung der intraoperativen Blutungsmenge erfolgt in der Regel durch die Messung des verlorenen Blutes aus dem Operationsbereich plus der Abschätzung des zusätzlichen aufgefangenen Blutes in Tupfern und Tüchern. Es hat sich jedoch erwiesen, dass die visuelle Einschätzung des Blutverlusts in Tupfern und Tüchern fast immer von einer starken Unter- oder Überschätzung [5] begleitet ist. Alternativen wie gravimetrische Messungen oder die Hämatin-Methode, die ursprünglich für die Quantifizierung der Regelblutung entwickelt wurde, scheinen sinnvoll, obwohl unterschiedliche Ergebnisse berichtet wurden [6, 7]. Mindestens in der gynäkologischen Chirurgie wurde die Hämatin-Methode klinisch validiert [8]. Insgesamt scheinen jedoch die einfacheren und schnelleren gravimetrischen Methoden für den Einsatz in der täglichen Praxis aktuell am praktikabelsten [9], wenn die Quantifizierung des Blutverlusts durch Messung in Sammelgefäßen plus Abschätzung des Verlusts in Tüchern zu ungenau ist.

\section{Unterschiedliche pathophysiologische Hintergründe von Koagulopathien}

Wie bereits eingangs erwähnt, besteht eine gewisse Schwierigkeit, objektiv zwischen einer Blutung, die primär durch eine Gefäßverletzung entsteht, und einer Blutung, die primär 
auf einer Koagulopathie beruht, zu unterscheiden. Dennoch ist eine solche Unterscheidung auf dem Boden des klinischen Bildes wichtig, da sich aus unterschiedlichen Pathophysiologien logischerweise unterschiedliche Interventionsstrategien ergeben. Blutungen, die primär durch die Verletzungen von Gefäßen zustande kommen, sind umschrieben, und das Volumen der Blutung steht mit dem Durchmesser des verletzten Gefäßes in direktem Zusammenhang.

Im Gegensatz dazu ist die koagulopathische Blutung diffuser Natur und daher bei typischer Ausprägung an verschiedenen Stellen im selben Patienten zu beobachten, solange die Gerinnungsstörung anhält. In den letzten Jahren hat sich herauskristallisiert, dass es unterschiedliche Arten von Koagulopathien gibt, die zu intra- oder perioperativen Blutungen führen. So kennen wir mittlerweile (zum Teil grundlegende) Unterschiede bei der klassischen disseminierten intravasalen Koagulopathie (DIC), der davon abzugrenzenden Traumainduzierten Koagulopathie, der Verdünnungskoagulopathie, den Koagulopathien bei geburtshilflichen Komplikationen, in der Herz-Thorax-Chirurgie und bei Lebererkrankungen sowie der Koagulopathie bei orthopädischen und anderen viszeralchirurgischen Eingriffen. Das Wissen um die wahrscheinlichste Pathophysiologie des jeweiligen koagulopathischen Geschehens ist wichtig, da sich hieraus Hinweise für möglicherweise unterschiedliche therapeutische Ansätze ableiten lassen.

Die Verdünnungskoagulopathie ist ein seit Langem bekanntes, klinisch relevantes Phänomen, das vor allem bei Volumenersatz ohne Gerinnungsfaktoren zu beobachten ist [10]. Es kann allerdings auch bei der alleinigen Verwendung von Plasma (vor allem über längere Zeit) auftreten, da während der Produktion von gefrorenem Frischplasma (fresh frozen plasma (FFP)) ein Teil der Gerinnungsfaktoraktivität verloren geht; mit FFP alleine kann daher bei anzustrebender Isovolämie keine gegenüber dem Normalzustand isoaktive Konzentration an Gerinnungsfaktoren erreicht werden [11]. Es gibt einige Hinweise darauf, dass die Verdünnungskoagulopathie vor allem ein Problem der Fibrin-Vernetzung und der Fibrin-Stabilität ist, d.h. im Wesentlichen die Endstrecke der Gerinnungskaskade betrifft (Fibrinogen und Faktor XIII sowie Fibrinolyse) [12-14].

Geburtshilfliche Blutungskomplikationen können sich als Folge der abnormen Gerinnungsaktivierung innerhalb einer nicht infektiös bedingten DIC präsentieren [15], die durch den Einstrom von Thromboplastinen in das Gefäßsystem [16] entsteht. Eine verstärkte Fibrinolyse scheint die Austreibung der Plazenta nach der Geburt zu unterstützen und ist in der Regel - danach selbstlimitierend [17]; dies deutet darauf hin, dass in diesen Situationen die Aktivierung der Fibrinolyse eine physiologisch wie pathophysiologisch wichtige Rolle spielt.

Gerinnungsstörungen bei Trauma und Polytrauma haben in den letzten Jahren wieder vermehrte Aufmerksamkeit erhalten, da sich gezeigt hat, dass sich die Trauma-induzierte
Koagulopathie von der DIC unterscheidet. Es wird vermutet, dass die Koagulopathie des akuten Traumas (acute traumatic coagulopathy (ATC)) vor allem auf der endothelialen Aktivierung von Protein $\mathrm{C}$ als zentralem Mechanismus beruht; in der Folge kommt es zum raschen Auftreten einer eigentlichen Antikoagulation und Fibrinolyse [18, 19]. Neuere Arbeiten beurteilen das Ausmaß der klinischen und pathophysiologischen Überlappung aber durchaus kontrovers [20, 21], was belegt, dass hier zu einem tieferen Verständnis weitere Forschung notwendig ist.

Blutungen sind ein wichtiger Faktor für eine erhöhte Mortalität in der Herzchirurgie [22] und daher ein relativ gut untersuchtes Phänomen, was zur Aufdeckung verschiedener pathophysiologischer Prozesse (wie z.B. Störung der normalen endothelialen Regulation bei Einführung von Fremdoberflächen [23]) führte. Erst weitere Untersuchungen werden den besten Weg zur Risikostratifizierung und Reduktion von Blutungskomplikationen ebnen [24, 25]. Die positiven Ergebnisse der Studien zum Einsatz von Antifibrinolytika erlauben aber mindestens die Erkenntnis, dass die Regulation der Fibrinolyse einen erheblichen Einfluss auf das klinische Ergebnis [26] hat.

Chirurgische Interventionen bei Patienten mit chronischen Lebererkrankungen erfordern besondere Aufmerksamkeit, da diese Patienten oft periinterventionelle Unterstützung in der Vorbereitung auf die Operationen benötigen; dies, da die Leber häufig bereits in ihrer Fähigkeit zur Synthese von Gerinnungsfaktoren eingeschränkt ist, was prognostisch bedeutsam ist [27]. Die aktive Vorbereitung solcher Patienten, inklusive der Verbesserung der fibrinolytischen Dysbalance, kann helfen, Komplikationen zu reduzieren [28]. Mittelschwere bis schwere Thrombozytopenien bei diesen Patienten wurden lange als Ausdruck eines erhöhten Milz-Poolings bei veränderten Flussvolumina im Pfortadergebiet interpretiert, jedoch deutet die Entdeckung des linienspezifischen Zytokins Thrombopoietin (TPO) auf eine direkte Verbindung zwischen hepatozellulärer Funktion und Thrombopoiese hin [29].

In der orthopädischen Chirurgie wird die lokale Blutstillung außerhalb der Weichteile durch die mechanischen Eigenschaften des Knochens, der keine Kompression zulässt, erschwert. Diese Problematik kann partiell durch «abdichtende» Maßnahmen umgangen werden [30]. Die Resultate von Interventionsstudien mit Antifibrinolytika erlauben wiederum abzuleiten, dass auch in diesem Patientengut die Regulierung der Fibrinolyse eine relevante Rolle spielt [31]. Nicht zu unterschätzen ist in diesem Zusammenhang auch die Wahl einer adäquaten perioperativen Thromboseprophylaxe [32, 33], da diese direkt mit intraoperativen Blutungen und anderen Outcome-Parametern wie der Länge des Krankenhausaufenthalts [34] assoziiert sind. Hier eröffnen neue Substanzen, deren Einsatz erst postoperativ begonnen werden muss [32, 33], neue Möglichkeiten.

In der viszeralen Chirurgie hat sich gezeigt, dass eine Optimierung mit einer Abnahme perioperativer Komplikationen, 
einschließlich Blutungen [35], assoziiert sein kann. Auch hier scheint die Wahl der adäquaten Thromboseprophylaxe wichtig, um Blutungskomplikationen gering zu halten [36]. Bei Patienten mit ansonsten unerklärter intraoperativer Koagulopathie gibt es Hinweise darauf, dass der relative Verlust der Vernetzungskapazität durch Verlust von Faktor XIII (im Vergleich zur Menge an gebildetem Prothrombin) zu einem frühen Verlust der Gerinnselfestigkeit und damit zu einem erhöhten Blutverlust [2, 37] führt. Unsere Erfahrungen zeigen, dass der perioperative, erworbene Faktor-XIII-Mangel ein häufiges Phänomen ist [38]. Dies ist wichtig, da Faktor XIII Fibrinogen vor der Fibrinogenolyse schützen kann, da er Antiplasmin an Fibrinogen bindet [39], was klinisch relevant zu sein scheint [40].

\section{Optionen zur Behandlung perioperativer koagulopathischer Blutungen}

Auf den ersten Blick scheint es logisch, dass die beste Option für die Ersatztherapie bei der Behandlung relevanter perioperativer koagulopathischer Blutungen die Verwendung von Vollblut ist [41-43]. Diese Vorgehensweise ist immer noch von Vorteil in Situationen, in denen keine ausreichenden Vorräte an labilen Blutprodukten vorhanden sind [4446]. Die Standardtherapie ist heutzutage jedoch die Komponententherapie, sei es durch den Einsatz labiler (zelluläre Komponenten, FFP) oder stabiler Komponenten (lyophilisierte Faktorkonzentrate).

Die wesentliche Herausforderung besteht heute vor allem darin zu entscheiden, wie man am besten mit einer perioperativen Gerinnungsstörung angesichts des Mangels an prospektiven, kontrollierten Studien in diesem Bereich umgeht. Die folgenden Ausführungen stellen daher die Überlegungen des Autors dar, basierend auf publizierten Resultaten und einer mehr als 20-jährigen Erfahrung im perioperativen Hämostasemanagement in einem Krankenhaus der Maximalversorgungsstufe.

Der gegenwärtige Wissensstand legt nahe, dass eine bestehende Verdünnungskoagulopathie nicht mit FFP alleine überwunden werden kann, da die Produktion von FFP von einem definierten Aktivitätsverlust pro Volumeneinheit begleitet wird. Daher wird eine bereits bestehende Verdünnung von Gerinnungsfaktoren durch den Gebrauch von FFP alleine nicht komplett kompensiert [11]. Bei größeren orthopädischen Eingriffen haben kolloidale Flüssigkeiten einen stärkeren dilutionskoagulopathischen Effekt auf die Gerinnselfestigkeit als Kristalloide; dieser Effekt kann teilweise durch die Gabe von Fibrinogen überwunden werden. Dies hat jedoch keinen Einfluss auf die Notwendigkeit einer Erythrozytentransfusion [47]. Thrombelastographische In-vitro-Experimente haben gezeigt, dass die Zugabe von Fibrinogen vor allem die durch Kristalloide induzierte Verminderung der Gerinnselfestigkeit verbessert, während Faktor XIIIa vor allem den Verlust an Gerinnselfestigkeit durch Kolloide [48] verbessert.

Patienten mit «klassischer» DIC können mittels hämostatischer Therapie niemals kausal behandelt werden. Die einzige Möglichkeit der kausalen Therapie ist die Behandlung der Ursache für die DIC. Allerdings kann die Behandlung der Gerinnungsstörung eine große Hilfe sein, um ein ausreichend großes Zeitfenster für eine kausale Therapie zu schaffen. $\mathrm{Zu}$ diesem Zweck scheint es wichtig, zwei wesentliche Regeln zu berücksichtigen:

- eine überschießende Thrombin-Bildung zu verhindern bzw. den bestehenden Einfluss von Thrombin zu reduzieren; insbesondere dann, wenn eine Blutung nicht im Vordergrund steht [49], und

- verbrauchte Gerinnungskomponenten adäquat zu ersetzen, um eine ausreichende Fibrin-Bildung sicherzustellen [50].

Um eine überschießende Thrombin-Bildung zu verhindern bzw. einen bestehenden Thrombin-Einfluss zu reduzieren, empfehlen die meisten Fallberichte [51-56] oder prospektive Studien $[57,58]$ die Verwendung von Heparin oder Heparinoiden, solange keine relevanten Blutungen vorliegen. Darüber hinaus gibt es prospektive Studien sowie Erfahrungen aus Fallberichten, die darauf hindeuten, dass lösliches Thrombomodulin bei der Kontrolle einer «klassischen» DIC [59-65] hilfreich sein kann.

Bei geburtshilflichen Patienten hängen die Behandlungsmöglichkeiten, einmal mehr, von der zugrunde liegenden $\mathrm{Pa}-$ thophysiologie ab. Bei Patienten mit Fruchtwasserembolie und ausgeprägter Gerinnungsaktivierung, Verbrauch von Gerinnungsfaktoren und koagulopathischer Blutung ist das wichtigste Ziel, den Verlust der zirkulierenden Gerinnungsfaktoren zu reduzieren und sie bei Bedarf zu ersetzen. Angesichts der erhöhten fibrinolytischen Aktivität rund um die Geburt scheint die Verwendung von Tranexamsäure, die bereits relativ früh beschrieben wurde [66], hilfreich [67] und hat daher kürzlich erneut Aufmerksamkeit erhalten [68, 69]. Dieser Ansatz wird gegenwärtig in einer großen randomisierten, prospektiven, multizentrischen Studie [70] getestet. Allerdings ist bei klassischer DIC mit manifestem oder drohendem Multiorganversagen - was auch in der Geburtshilfe auftreten kann - diesbezüglich aufgrund von Tierversuchen ein theoretischer Vorbehalt anzumelden [71]; die oben genannte Studie dürfte hier Klärung bringen. Bei elektiver Schnittentbindung reduziert Tranexamsäure den Blutverlust [68]. Zusätzlich zu der Modulation der Fibrinolyse - und angesichts der großen Wundfläche bei hämorrhagischen postpartalen Patientinnen - muss eine ausreichende Substitutionstherapie vorbereitet sein, um den hohen Blutverlust zeitgerecht ausgleichen zu können. Wie oben erläutert, ist es unwahrscheinlich, eine bestehende, relevante (Verdünnungs-)Koagulopathie mit FFP alleine bekämpfen zu können. Daher scheint die Ersatztherapie mit Gerinnungsfaktorkonzentraten aktuell der beste Behandlungsansatz zu sein. Optimal wäre für die diesbezügliche Entscheidungsfindung die Messung spezifi- 
scher Gerinnungsfaktoraktivitäten; diese benötigt aber in einer solchen Situation, in der ein rasches Vorgehen nötig ist, häufig vergleichsweise viel Zeit, wenn auch in einzelnen Krankenhäusern kurze Rückmeldezeiten von 40 min erreicht werden können. Die Verwendung viskoelastischer Verfahren erlaubt aber wenigstens die rasche Identifikation einer Koagulopathie (wenn auch nicht die Feststellung eines spezifischen Gerinnungsfaktormangels [72-74]). Es ist daher wichtig, einen Algorithmus definiert zu haben (siehe z.B. [75]), der das Vorgehen während des Behandlungsprozesses auf der Grundlage der klinischen Situation definiert und alle potenziellen Behandlungsmöglichkeiten (pharmakologische Therapien, invasive Eingriffe einschließlich Embolisation, chirurgische Eingriffe) berücksichtigt. Die Fibrinogeninfusion bei Patientinnen mit postpartalen Blutungen (postpartum hemorrhage (PPH)) hat kürzlich große Aufmerksamkeit erhalten, da eine präpartale Fibrinogenkonzentration $>4 \mathrm{~g} / \mathrm{l}$ in einer Studie einen hohen $(79 \%)$ negativ prädiktiven Wert hatte, was darauf hindeutet, dass sehr hohe Fibrinogenspiegel über $4 \mathrm{~g} / \mathrm{l}$ einen relevanten Schutzeffekt gegenüber einer schweren PPH haben könnten [76]. Allerdings wurden widersprüchliche Ergebnisse in anderen Populationen gefunden [77], was zur Planung einer prospektiven, randomisierten, kontrollierten Studie führte [78].

Die Behandlung von Gerinnungsstörungen bei ATC-Patienten hat ebenfalls neues Interesse geweckt, was damit zusammenhängen dürfte, dass neuere Forschungsergebnisse darauf hinweisen, dass die Pathophysiologien von ATC und DIC unterschiedlich sind. Darüber hinaus hat die Entwicklung von thrombelastographischen Techniken erlaubt, quantitative Informationen über den Gerinnungsprozess quasi begleitend während der frühen Stadien der Behandlung [79] zu erhalten. Im Gegensatz zu Daten aus der elektiven Chirurgie sollte es die Differentialdiagnose der beobachteten Veränderungen $[19,80]$ hoffentlich erlauben, eine zielgerichtete Therapie zu betreiben; prospektive Studien, die einen klinischen Endpunkt untersuchen, stehen noch aus. Eine FibrinogenErsatztherapie hat auch in der Behandlung von Trauma-Patienten Aufmerksamkeit erhalten [81], da es offensichtlich zu sein scheint, dass niedrige Fibrinogenspiegel ein Prädiktor für Morbidität und Mortalität sind [82]. Ob es sich dabei um einen Surrogat-Marker oder einen kausalen Zusammenhang handelt, bleibt noch zu klären. Die Ersatztherapie, die bei Kohorten einzelner Zentren erfolgreich eingesetzt wird [8387], sollte in prospektiven randomisierten Studien validiert werden.

Die Verwendung von Antifibrinolytika/Tranexamsäure hat sich bei orthopädischen Patienten als vorteilhaft herausgestellt - sowohl bei systemischer als auch bei topischer Verwendung [88-96]; ihre Anwendung wird daher empfohlen [97-99]. Wenn eine übermäßige Blutung nicht mit dem (prophylaktischen) Einsatz von Antifibrinolytika (verhindert oder) kontrolliert werden kann, sollten zunächst wieder die bereits oben erwähnten Argumente zur Dilutionskoagulopa- thie beachtet werden [11-14], um ausreichend hohe Gerinnungsfaktorkonzentrationen während und nach orthopädischen Operationen sicherzustellen. Es ist noch zu klären, ob es in diesem Setting zu einem generellen Verlust von Gerinnungsfaktoren kommt oder ob einzelne, spezifisch erworbene Anomalien identifiziert werden können, wie z.B. Mangel an von-Willebrand-Faktor [100, 101], Fibrinogen [102] und Faktor XIII [38, 103, 104].

Wie in den anderen Disziplinen ist auch in der Viszeralchirurgie die Identifizierung der zugrunde liegenden Pathologie entscheidend für die Auswahl der adäquaten Therapie. Wenn die Leberfunktion z.B. aufgrund einer Zirrhose stark beeinträchtigt ist, so sollte man sich des Ungleichgewichts zwischen Fibrinolyse und prokoagulanter Aktivität bewusst sein, was die Verwendung von Antifibrinolytika zusammen mit dem Ersatz von Gerinnungsfaktoren nahelegt. Bisherige Erfahrungen legen nahe, dass dafür am effektivsten PPSB (Prothrombinkonzentrat) verwendet wird [105]. Wenn hierfür FFP eingesetzt wird, so sollte auf die Verwendung einer ausreichend großen Menge geachtet werden (20-30 ml/kg Körpergewicht, d.h. ca. 1,5-2 1 FFP bei 70 kg Körpergewicht [106]; dementsprechend muss auf eine mögliche Volumenüberladung geachtet werden). Darüber hinaus kann - bei Thrombopenie - eine Thrombozytentransfusion sinnvoll sein; angesichts eines möglichen Milz-Pooling-Effekts sollte die Transfusion (wenn präoperativ keine Blutung besteht) erst unmittelbar vor oder während der Operation gegeben werden. Da diese Patienten nicht selten verminderte TPOSpiegel zeigen, kann in spezifischen, elektiven Fällen (d.h. bei Patienten, die sonst nicht zu managen wären) präoperativ die Optimierung unter Einsatz eines TPO-Agonisten in $\mathrm{Zu}$ sammenarbeit mit einem Hämatologen in Betracht gezogen werden [107], wobei allerdings auch die potenzielle Toxizität der Substanzen in Betracht zu ziehen ist [108]. Zu Patienten mit viszeralen (vor allem gastrointestinalen) Operationen ohne spezifische Lebererkrankung gibt es nur wenige prospektive Studien, die potenzielle Behandlungsoptionen bei koagulopathischen Blutungen während der Operation untersucht haben. Unsere früheren Arbeiten zur entsprechenden Pathophysiologie haben einen erworbenen Mangel der Quervernetzungskapazität durch Faktor XIII (erworbener FaktorXIII-Mangel) als häufigen Grund für den deutlich erhöhten Verlust von Gerinnselfestigkeit bei diesen Patienten identifiziert [2]. In der Folge konnten wir in einer prospektiven, randomisierten, doppelblinden, placebokontrollierten Studie zeigen, dass die Substitution von Faktor XIII früh während der Operation in dieser Patientenpopulation zur Vermeidung des Verlusts an Gerinnselfestigkeit, zur Verringerung des Verlusts von Fibrinogen und zur Verringerung des Blutverlusts führt [40]. Somit scheint dieses Konzept ein interessanter neuer Ansatz $[37,109]$ zu sein, den wir in unserem Haus in den letzten Jahren mit Erfolg angewendet haben (Manuskript in Vorbereitung). 


\section{Schlussfolgerung}

Zusammengefasst lassen die heute verfügbaren Daten zu peri- und intraoperativen Blutungskomplikationen den Schluss zu, dass diese Blutungen in unterschiedlichen Populationen auf unterschiedlichen Pathophysiologien beruhen und daher auch unterschiedliche Behandlungsstrategien benötigen. Allgemein kann festgehalten werden, dass viskoelastische Verfahren die frühe Feststellung einer Koagulopathie erlauben (wenn aktuell auch (noch?) nicht die Feststellung spezifischer Gerinnungsfaktordefizite) und dass eine konsequente, frühzeitige Behandlung einer Koagulopathie in der Regel die Ausbildung eines höhergradigen Geschehens verhindern oder mindestens relevant verzögern kann.

\section{Disclosure Statement}

Referenten- oder Beratertätigkeiten für CSL Behring, Baxter, Bayer, TEM und Haemonetics.

\section{Literatur}

1 Nuttall GA, Oliver WC, Santrach PJ, et al: Efficacy of a simple intraoperative transfusion algorithm for nonerythrocyte component utilization after cardiopulmonary bypass. Anesthesiology 2001;94:773781; discussion 5A-6A.

2 Wettstein P, Haeberli A, Stutz M, et al: Decreased factor XIII availability for thrombin and early loss of clot firmness in patients with unexplained intraoperative bleeding. Anesth Analg 2004;99:15641569

-3 Tanaka KA, Key NS, Levy JH: Blood coagulation: hemostasis and thrombin regulation. Anesth Analg 2009;108:1433-1446.

4 Schols SE, Feijge MA, Lance MD, et al: Effects of plasma dilution on tissue factor-induced thrombin generation and thromboelastography: partly compensating role of platelets. Transfusion 2008;48: 2384-2394.

$\checkmark 5$ Meiser A, Casagranda O, Skipka G, et al: Quantifizierung von Blutverlusten. Wie genau ist visuelles Schätzen und wovon hängt die Genauigkeit $a b$ ? Anaesthesist 2001;50:13-20.

6 Lee MH, Ingvertsen BT, Kirpensteijn J, et al: Quantification of surgical blood loss. Vet Surg 2006;35:388-393.

7 Johar RS, Smith RP: Assessing gravimetric estimation of intraoperative blood loss. J Gynecol Surg 1993;9:151-154.

8 Chua S, Ho LM, Vanaja K, et al: Validation of a laboratory method of measuring postpartum blood loss. Gynecol Obstet Invest 1998;46:31-33.

$\checkmark$ Schorn MN: Measurement of blood loss: review of the literature. J Midwifery Womens Health 2010; 55:20-27.

10 Murray DJ, Pennell BJ, Weinstein SL, et al Packed red cells in acute blood loss: dilutional coagulopathy as a cause of surgical bleeding. Anesth Analg 1995;80:336-342.

11 Jámbor C, Lesch V, Schnider TW, et al: Fresh frozen plasma is ineffective for reversal of coagulation factor dilution in an in vitro model. Appl Cardiopulm Pathophysiol 2009;13:130-133.

12 Fenger-Eriksen C, Tonnesen E, Ingerslev J, et al: Mechanisms of hydroxyethyl starch-induced dilutional coagulopathy. J Thromb Haemost 2009;7: 1099-1105.

13 Nielsen VG: Hydroxyethyl starch enhances fibrinolysis in human plasma by diminishing alpha2antiplasmin-plasmin interactions. Blood Coagul Fibrinolysis 2007:18:647-656.

14 Haas T, Fries D, Velik-Salchner C, et al: The in vitro effects of fibrinogen concentrate, factor XII and fresh frozen plasma on impaired clot formation after $60 \%$ dilution. Anesth Analg 2008;106:13601365 , table of contents.
15 Thachil J, Toh CH: Disseminated intravascular coagulation in obstetric disorders and its acute haematological management. Blood Rev 2009;23:167176.

16 Ben-Ami I, Fuchs N, Schneider D, et al: Coagulopathy associated with dilation and evacuation for second-trimester abortion. Acta Obstet Gynecol Scand 2012;91:10-15.

17 Hellgren M: Hemostasis during normal pregnancy and puerperium. Sem Thromb Hemost 2003;29: 125-130.

18 Davenport R: Pathogenesis of acute traumatic coagulopathy. Transfusion 2013;53(suppl 1):23S-27S

19 Schochl H, Frietsch T, Pavelka M, et al: Hyperfibrinolysis after major trauma: differential diagnosis of lysis patterns and prognostic value of thrombelastometry. J Trauma 2009;67:125-131.

20 Yanagida Y, Gando S, Sawamura A, et al: Normal prothrombinase activity, increased systemic thrombin activity, and lower antithrombin levels in patients with disseminated intravascular coagulation at an early phase of trauma: comparison with acute coagulopathy of trauma-shock. Surgery 2013; 154:48-57.

21 Johansson PI, Sorensen AM, Perner A, et al: Disseminated intravascular coagulation or acute coagulopathy of trauma shock early after trauma? An observational study. Crit Care 2011;15:R272.

22 Ranucci M, Baryshnikova E, Castelvecchio S, et al: Major bleeding, transfusions, and anemia: the deadly triad of cardiac surgery. Ann Thorac Surg 2013;96:478-485.

23 Achneck HE, Sileshi B, Parikh A, et al: Pathophysiology of bleeding and clotting in the cardiac surgery patient: from vascular endothelium to circulatory assist device surface. Circulation 2010;122: 2068-2077.

24 Yang L, Vuylsteke A, Gerrard C, et al: Postoperative fibrinogen level is associated with postoperative bleeding following cardiothoracic surgery and the effect of fibrinogen replacement therapy remains uncertain. J Thromb Haemost 2013;11:1519-1526.

25 Nielsen VG: Coagulation crystal ball: why can't we predict bleeding after cardiac surgery? Anesth Analg 2012;115:490-492.

26 Ozier Y, Schlumberger S: Pharmacological approaches to reducing blood loss and transfusions in the surgical patient. Can J Anaesth 2006;53(suppl): S21-29.

27 Esmat Gamil M, Pirenne J, Van Malenstein H, et al: Risk factors for bleeding and clinical implications in patients undergoing liver transplantation. Transplant Proc 2012;44:2857-2860.
28 Senzolo M, Burra P, Cholongitas E, et al: New insights into the coagulopathy of liver disease and liver transplantation. World J Gastroenterol 2006; 12:7725-7736.

29 Peck-Radosavljevic M: Thrombocytopenia in liver disease. Can J Gastroenterol 2000;14(suppl D): 60D-66D.

30 von Arx T, Jensen SS, Hanni S, et al: Haemostatic agents used in periradicular surgery: an experimental study of their efficacy and tissue reactions. Int Endod J 2006;39:800-808.

31 Vijay BS, Bedi V, Mitra S, et al: Role of tranexamic acid in reducing postoperative blood loss and transfusion requirement in patients undergoing hip and femoral surgeries. Saudi J Anaesth 2013;7:29-32.

32 Yoshida Rde A, Yoshida WB, Maffei FH, et al: Systematic review of randomized controlled trials of new anticoagulants for venous thromboembolism prophylaxis in major orthopedic surgeries, compared with enoxaparin. Ann Vasc Surg 2013; 27:355-369.

33 Oberweis BS, Nukala S, Rosenberg A, et al Thrombotic and bleeding complications after orthopedic surgery. Am Heart J 2013;165:427-433.e1.

34 Vera-Llonch M, Hagiwara M, Oster G: Clinical and economic consequences of bleeding following major orthopedic surgery. Thromb Res 2006;117: 569-577.

35 Abu Hilal M, Underwood T, Taylor MG, et al: Bleeding and hemostasis in laparoscopic liver surgery. Surg Endosc 2010;24:572-577.

36 Girard P, Demaria J, Lillo-Le Louet A, et al: Transfusions, major bleeding, and prevention of venous thromboembolism with enoxaparin or fondaparinux in thoracic surgery. Thromb Haemost 2011;106:1109-1116.

37 Korte W: Fibrinmonomer und Faktor XIII - Neues Konzept bei ungeklärter intraoperativer Blutungsneigung. Haemostaseologie 2006;26(suppl 1):S30-35.

38 Korte W, Hinnen C, Degiacomi P, et al: Relevant F. XIII deficiency in the surgical intensive care unit is more frequent than a relevant fibrinogen deficiency. Haemostaseologie 2007;27:A61.

39 Mosesson MW, Siebenlist KR, Hernandez I, et al: Evidence that alpha2-antiplasmin becomes covalently ligated to plasma fibrinogen in the circulation: a new role for plasma factor XIII in fibrinolysis regulation. J Thromb Haemost 2008;6:15651570.

40 Korte WC, Szadkowski C, Gahler A, et al: Factor XIII substitution in surgical cancer patients at high risk for intraoperative bleeding. Anesthesiology 2009;110:239-245. 
41 Manno CS, Hedberg KW, Kim HC, et al: Comparison of the hemostatic effects of fresh whole blood, stored whole blood, and components after open heart surgery in children. Blood 1991;77:930-936.

42 Laine E, Steadman R, Calhoun L, et al: Comparison of RBCs and FFP with whole blood during liver transplant surgery. Transfusion 2003;43:322-327.

43 Gruenwald CE, McCrindle BW, Crawford-Lean L, et al: Reconstituted fresh whole blood improves clinical outcomes compared with stored component blood therapy for neonates undergoing cardiopulmonary bypass for cardiac surgery: a randomized controlled trial. J Thorac Cardiovasc Surg 2008;136:1442-1449.

44 Brown HR Jr: The distribution and use of human whole blood in the Pacific war. U S Nav Med Bull 1947;47:396-420.

45 Daban JL, Kerleguer A, Clavier B, et al: Fresh whole blood transfusion for war surgery: the experience of the Kabul French combat support hospital from 2006 to 2009 (article in French). Ann Fr Anesth Reanim 2012;31:850-856.

46 Robertson LB: The transfusion of whole blood: a suggestion for its more frequent employment in war surgery. Br Med J 1916;2:38-40.

47 Mittermayr M, Streif W, Haas T, et al: Hemostatic changes after crystalloid or colloid fluid administration during major orthopedic surgery: the role of fibrinogen administration. Anesth Analg 2007;105: 905-917, table of contents.

48 Nielsen VG: Colloids decrease clot propagation and strength: role of factor XIII-fibrin polymer and thrombin-fibrinogen interactions. Acta Anaesthesiol Scand 2005;49:1163-1171.

49 Wada H, Asakura H, Okamoto K, et al: Expert consensus for the treatment of disseminated intravascular coagulation in Japan. Thromb Res 2010; 125:6-11.

50 Di Nisio M, Baudo F, Cosmi B, et al: Diagnosis and treatment of disseminated intravascular coagulation: guidelines of the Italian Society for Haemostasis and Thrombosis (SISET). Thromb Res 2012; 129:e177-184.

51 Perry JH, Lazar HL, Quillen K, et al: Successful longterm management of aneurysm-associated chronic disseminated intravascular coagulation with low molecular weight heparin. J Card Surg 2012;27:730-735.

52 Gomes OM, Gomes ES: Dipyridamole and low doses of heparin as a new successful physiopathologic and therapeutic approach in 2 cases of disseminated intravascular coagulation. Heart Surg Forum 2010;13:E49-51.

53 Togami K, Nagai Y, Arima H, et al: Successful treatment of chronic disseminated intravascular coagulation syndrome with continuous subcutaneous infusion of heparin using a mobile infusion pump: report of 2 cases (article in Japanese). Rinsho Ketsueki 2009;50:1700-1705.

54 Miyahara S, Yasu T, Yamada Y, et al: Subcutaneous injection of heparin calcium controls chronic disseminated intravascular coagulation associated with inoperable dissecting aortic aneurysm in an outpatient clinic. Intern Med 2007;46:727-732.

55 Garcia Fernandez JR, Lopez Berenguel F, Ais C: Long-term treatment with low molecular weight heparin, of chronic disseminated intravascular coagulation (article in Spanish). An Med Interna 2003;20:191-194.

56 Kurihara I, Yamaguchi Y, Soma J, et al: Two aged patients with chronic renal failure and chronic disseminated intravascular coagulation secondary to aortic aneurysms: effect of continuous subcutaneous heparin infusion therapy (article in Japanese) Nihon Jinzo Gakkai Shi 2000;42:603-607.
57 Sakuragawa N, Hasegawa H, Maki M, et al: Clinical evaluation of low-molecular-weight heparin (FR-860) on disseminated intravascular coagulation (DIC) - a multicenter co-operative doubleblind trial in comparison with heparin. Thromb Res 1993;72:475-500.

58 Cofrancesco E, Boschetti C, Leonardi P, et al: Dermatan sulphate for the treatment of disseminated intravascular coagulation (DIC) in acute leukemia: a randomised, heparin-controlled pilot study. Thromb Res 1994;74:65-75.

59 Iyama S, Sato T, Murase K, et al: Intermittent administration of recombinant human soluble thrombomodulin successfully controlled chronic disseminated intravascular coagulation in a patient with dissecting aortic aneurysm on an outpatient basis. Blood Coagul Fibrinolysis 2012;23:548-550.

60 Kawano N, Yoshida S, Ono N, et al: Clinical features and outcomes of 35 disseminated intravascular coagulation cases treated with recombinant human soluble thrombomodulin at a single institution. J Clinical Exp Hematop 2011;51:101-107.

61 Aikawa N, Shimazaki S, Yamamoto Y, et al: Thrombomodulin alfa in the treatment of infectious patients complicated by disseminated intravascular coagulation: subanalysis from the phase 3 trial. Shock 2011;35:349-354.

62 Uni M, Yoshimi A, Maki H, et al: Successful treatment with recombinant thrombomodulin for B-cell lymphoma-associated hemophagocytic syndrome complicated by disseminated intravascular coagulation. Int J Clin Exp Pathol 2013;6:1190-1194.

63 Masuda S, Adachi O, Ito K, et al: Successful treatment using recombinant thrombomodulin for disseminated intravascular coagulation associated with recurrent prosthetic valve endocarditis (article in Japanese). Kyobu Geka 2013;66:101-105.

64 Kawano N, Kuriyama T, Yoshida S, et al: Clinical features and treatment outcomes of six patients with disseminated intravascular coagulation resulting from acute promyelocytic leukemia and treated with recombinant human soluble thrombomodulin at a single institution. Intern Med 2013;52:55-62.

65 Yagasaki H, Kato M, Shimozawa K, et al: Treatment responses for disseminated intravascular coagulation in 25 children treated with recombinant thrombomodulin: a single institution experience. Thromb Res 2012;130:e289-293.

66 Walzman M, Bonnar J: Effects of tranexamic acid on the coagulation and fibrinolytic systems in pregnancy complicated by placental bleeding. Arch Toxicol Suppl 1982;5:214-220.

67 Ferrer P, Roberts I, Sydenham E, et al: Anti-fibrinolytic agents in post partum haemorrhage: a systematic review. BMC Pregnancy Childbirth 2009;9: 29.

68 Peitsidis P, Kadir RA: Antifibrinolytic therapy with tranexamic acid in pregnancy and postpartum. Expert Opin Pharmacother 2011;12:503-516.

69 Bonnet MP, Tesniere A, Mignon A: Transfusion for post-partum haemorrhage: what's new in 2011? (article in French). Transfus Clin Biol 2011;18:129-132.

70 Shakur H, Elbourne D, Gulmezoglu M, et al: The WOMAN Trial (World Maternal Antifibrinolytic Trial): tranexamic acid for the treatment of postpartum haemorrhage: an international randomised, double blind placebo controlled trial. Trials 2010; 11:40.

71 Asakura H, Sano Y, Yoshida T, et al: Beneficial effect of low-molecular-weight heparin against lipopolysaccharide-induced disseminated intravascular coagulation in rats is abolished by coadministration of tranexamic acid. Intensive Care Med 2004:30:1950-1955.
2 Rugeri L, Levrat A, David JS, et al: Diagnosis of early coagulation abnormalities in trauma patients by rotation thrombelastography. J Thromb Haemost 2007;5:289-295.

73 Jambor C, Reul V, Schnider TW, et al: In vitro inhibition of factor XIII retards clot formation, reduces clot firmness, and increases fibrinolytic effects in whole blood. Anesth Analg 2009;109:10231028.

74 Tauber H, Innerhofer P, Breitkopf R, et al: Prevalence and impact of abnormal ROTEM ${ }^{\circledR}$ assays in severe blunt trauma: results of the 'Diagnosis and Treatment of Trauma-Induced Coagulopathy (DIA-TRE-TIC) study'. Br J Anaesth 2011;107: 378-387.

75 Irita K, Inada E: Guidelines for management of critical bleeding in obstetrics (article in Japanese). Masui 2011;60:14-22.

76 Charbit B, Mandelbrot L, Samain E, et al: The decrease of fibrinogen is an early predictor of the severity of postpartum hemorrhage. J Thromb Haemost 2007;5:266-273.

77 Peyvandi F, Biguzzi E, Franchi F, et al: Elevated prepartum fibrinogen levels are not associated with a reduced risk of postpartum hemorrhage. J Thromb Haemost 2012;10:1451-1453.

78 Wikkelsoe AJ, Afshari A, Stensballe J, et al: The FIB-PPH trial: fibrinogen concentrate as initial treatment for postpartum haemorrhage: study protocol for a randomised controlled trial. Trials 2012; 13:110.

79 Carroll RC, Craft RM, Langdon RJ, et al: Early evaluation of acute traumatic coagulopathy by thrombelastography. Transl Res 2009;154:34-39.

80 Cotton BA, Harvin JA, Kostousouv V, et al: Hyperfibrinolysis at admission is an uncommon but highly lethal event associated with shock and prehospital fluid administration. J Trauma Acute Care Surg 2012;73:365-370; discussion 370.

81 Sorensen B, Larsen OH, Rea CJ, et al: Fibrinogen as a hemostatic agent. Semin Thromb Hemost 2012;38:268-273.

82 Inaba K, Karamanos E, Lustenberger T, et al: Impact of fibrinogen levels on outcomes after acute injury in patients requiring a massive transfusion. J Am Coll Surg 2013;216:290-297.

83 Schochl H, Posch A, Hanke A, et al: High-dose fibrinogen concentrate for haemostatic therapy of a major trauma patient with recent clopidogrel and aspirin intake. Scand J Clin Lab Invest 2010;70: 453-457.

84 Schochl H, Nienaber U, Hofer G, et al: Goal-directed coagulation management of major trauma patients using thromboelastometry (ROTEM)-guided administration of fibrinogen concentrate and prothrombin complex concentrate. Crit Care 2010;14:R55.

85 Schochl H, Forster L, Woidke R, et al: Use of rotation thromboelastometry (ROTEM) to achieve successful treatment of polytrauma with fibrinogen concentrate and prothrombin complex concentrate. Anaesthesia 2010;65:199-203.

86 Fries D, Martini WZ: Role of fibrinogen in traumainduced coagulopathy. Br J Anaesth 2010;105:116121.

87 Innerhofer P, Westermann I, Tauber H, et al: The exclusive use of coagulation factor concentrates enables reversal of coagulopathy and decreases transfusion rates in patients with major blunt trauma. Injury 2013;44:209-216.

88 Kagoma YK, Crowther MA, Douketis J, et al: Use of antifibrinolytic therapy to reduce transfusion in patients undergoing orthopedic surgery: a systematic review of randomized trials. Thromb Res 2009; 123:687-696. 
89 Elwatidy S, Jamjoom Z, Elgamal E, et al: Efficacy and safety of prophylactic large dose of tranexamic acid in spine surgery: a prospective, randomized, double-blind, placebo-controlled study. Spine 2008; 33:2577-2580.

90 Llau Pitarch JV, Diaz Alvarez A, Polonio Enriquez F, et al: Reduction of blood transfusion need with aprotinin in orthopedic surgery. Spanish Study Group on the Use of Aprotinin in Hip Arthroplasty (GEEEAAC) (article in Spanish). Rev Esp Anestesiol Reanim 2000;47:309-316.

91 Georgiadis AG, Muh SJ, Silverton CD, et al: A prospective double-blind placebo controlled trial of topical tranexamic acid in total knee arthroplasty. J Arthroplasty 2013;28(suppl):78-82.

92 Irisson E, Hemon Y, Pauly V, et al: Tranexamic acid reduces blood loss and financial cost in primary total hip and knee replacement surgery. Orthop Traumatol Surg Res 2012;98:477-483.

93 Eschen CT, Tengberg PT, Husted H, et al: Tranexamic acid reduces blood loss after major elective orthopaedic operations (article in Danish). Ugeskr Laeger 2012;174:47-49.

94 Sepah YJ, Umer M, Ahmad T, et al: Use of tranexamic acid is a cost effective method in preventing blood loss during and after total knee replacement. J Orthop Surg Res 2011;6:22

95 Gill JB, Chin Y, Levin A, et al: The use of antifibrinolytic agents in spine surgery. A meta-analysis. J Bone Joint Surg Am 2008;90:2399-2407.
96 Krohn CD, Sorensen R, Lange JE, et al: Tranexamic acid given into the wound reduces postoperative blood loss by half in major orthopaedic surgery. Eur J Surg Suppl 2003;(588):57-61.

97 Bruce W, Campbell D, Daly D, et al: Practical recommendations for patient blood management and the reduction of perioperative transfusion in joint replacement surgery. ANZ J Surg 2013;83: 222-229.

98 McCormack PL: Tranexamic acid: a review of its use in the treatment of hyperfibrinolysis. Drugs 2012;72:585-617.

99 Eubanks JD: Antifibrinolytics in major orthopaedic surgery. J Am Acad Orthop Surg 2010;18:132138.

100 Kroonen LT, Gillingham BL, Provencher MT: Orthopedic manifestations and management of patients with von Willebrand disease. Orthopedics 2008;31:263-267; quiz 268-269.

101 de Lange DW, Fijnheer R, Wittebol S: Acquired von Willebrand syndrome (article in Dutch). Ned Tijdschr Geneeskd 2003;147:1808-1811.

102 Carling MS, Jeppsson A, Wessberg P, et al: Preoperative fibrinogen plasma concentration is associated with perioperative bleeding and transfusion requirements in scoliosis surgery. Spine 2011; 36:549-555.
103 Kanda A, Kaneko K, Obayashi O, et al: The massive bleeding after the operation of hip joint surgery with the acquired haemorrhagic coagulation factor XIII(13) deficiency: two case reports. Case Rep Orthop 2013;2013:473014.

104 Nourbakhsh E, Anvari R, D’Cunha N, et al: Postoperative bleeding in a patient with normal screening coagulation tests. Am J Med Sci 2011; 342:262-264.

105 Lorenz R, Kienast J, Otto U, et al: Efficacy and safety of a prothrombin complex concentrate with two virus-inactivation steps in patients with severe liver damage. Eur J Gastroenterol Hepatol 2003;15:15-20.

106 Chowdhury P, Saayman AG, Paulus U, et al: Efficacy of standard dose and $30 \mathrm{ml} / \mathrm{kg}$ fresh frozen plasma in correcting laboratory parameters of haemostasis in critically ill patients. Br J Haematol 2004;125:69-73.

107 Aguilar C: Potential usefulness of thrombopoietin receptor agonists in haemophiliacs with thrombocytopaenia due to chronic liver disease. Blood Coagul Fibrinolysis 2013;24:231-236.

108 Afdhal NH, Giannini EG, Tayyab G, et al: Eltrombopag before procedures in patients with cirrhosis and thrombocytopenia. N Engl J Med 2012;367:716-724.

109 Korte W: F. XIII in perioperative coagulation management. Best Pract Res Clin Anaesthesiol 2010;24:85-93 